\title{
Elevated midline head positioning of extremely low birth weight infants: effects on cardiopulmonary function and the incidence of periventricular-intraventricular hemorrhage
}

\author{
Michael Kochan ${ }^{1,4} \cdot$ Bianca Leonardi $^{1,5} \cdot$ Angela Firestine $^{1,6} \cdot$ Jacob McPadden $^{1,7} \cdot$ Danielle Cobb $^{1,8}$. \\ Tushar A. Shah ${ }^{2} \cdot$ Turaj Vazifedan ${ }^{3} \cdot$ W. Thomas Bass $\mathbb{1 D}^{3}$
}

Received: 31 May 2018 / Revised: 19 September 2018 / Accepted: 5 October 2018 / Published online: 22 October 2018

(c) Springer Nature America, Inc. 2018

\begin{abstract}
Objective: Changes in cerebrovascular hemodynamics associated with head position may be important in the pathogenesis of periventricular-intraventricular hemorrhage (PIVH) in premature infants. This study evaluated the effect of elevated midline head positioning on cardiopulmonary function and the incidence of PIVH.

Study design: ELBW infants were randomized to FLAT (flat, supine) or ELEV (supine, bed elevated 30 degrees) for $96 \mathrm{~h}$. Cardiopulmonary function, complications of prematurity, and the occurrence of PIVH were documented.

Results: Infants were randomized into FLAT $(n=90)$ and ELEV groups $(n=90)$. No significant differences were seen in the incidence of BPD or other respiratory complications. The ELEV group developed significantly fewer grade 4 hemorrhages $(p=0.036)$ and survival to discharge was significantly higher in the ELEV group $(p=0.037)$.

Conclusions: Managing ELBW infants in an elevated midline head position for the first 4 days of life appears safe and may decrease the likelihood of severe PIVH and improve survival.
\end{abstract}

\section{Introduction}

$\triangle \mathrm{W}$. Thomas Bass

Thomas.Bass@chkd.org

1 Department of Pediatrics, Children's Hospital of The King's Daughters, Eastern Virginia Medical School, Norfolk, USA

2 Division of Neonatal-Perinatal Medicine, Department of Pediatrics, Children's Hospital of The King's Daughters, Eastern Virginia Medical School, Norfolk, USA

3 Division of Biostatistics, Department of Pediatrics, Children's Hospital of The King's Daughters, Eastern Virginia Medical School, Norfolk, USA

4 Present address: Division of Neonatology, University of Maryland School of Medicine, Baltimore, MD, USA

5 Present address: Pediatrix Medical Group of Ohio, Akron, $\mathrm{OH}$, USA

6 Present address: Central Ohio Newborn Medicine, Columbus, $\mathrm{OH}$, USA

7 Present address: Division of Neonatal-Perinatal Medicine, Yale University School of Medicine, New Haven, CT, USA

8 Present address: Arnold Palmer Hospital for Children, Orlando, FL, USA
The incidence of periventricular-intraventricular hemorrhage (PIVH) in preterm infants has decreased over the past several years with the advent of antenatal steroid administration and improvements in neonatal care. However, the incidence of PIVH in extremely low birth weight (ELBW) infants remains high. The incidence of PIVH is inversely proportional to gestational age, and hence the burden of PIVH related complications such as cerebral palsy, hydrocephalus, seizures and neurodevelopmental disability is greater in the ELBW population [1].

The pathogenesis of PIVH is multifactorial due to the intrinsic vulnerability of immature cerebral germinal matrix vessels subjected to changes in cerebral blood flow [2]. PIVH originates in the germinal matrix, a highly vascularized area of neuronal-glial precursor cells, most prominent in the developing brain from 22-32 weeks of gestation [1]. Regardless of gestational age, $90 \%$ of PIVH occurs in the first 3 days of life [1]. Significant fluctuations in cerebral blood flow, increases in cerebral venous pressure, and impaired autoregulation have been reported in ELBW premature infants with respiratory distress syndrome, the group of patients at greatest risk for PIVH [3]. Routine procedures 
Fig. 1 a shows the custom wedge frame designed the hold the bed at 30 degrees of elevation as seen in $\mathbf{b}, \mathbf{c}$ shows an ELBW manikin in the ELEV position
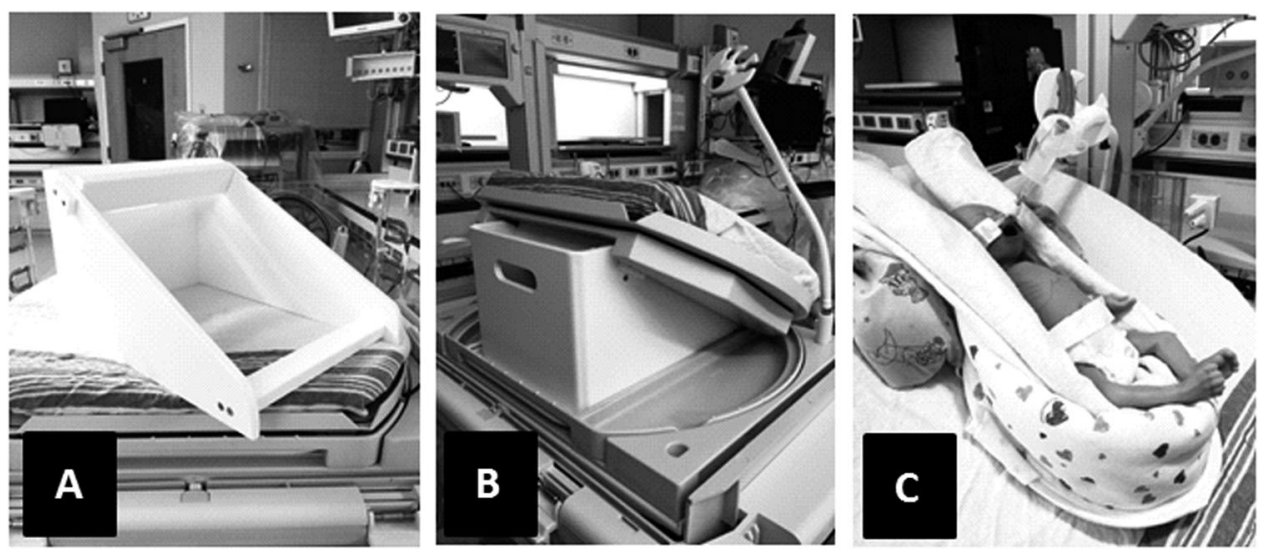

in critically ill newborns such as endotracheal tube adjustment, suctioning, repositioning of the infant, and even diaper changes have been associated with major cerebral circulatory fluctuations $[4,5]$.

Position associated changes in cerebral blood flow are enhanced in ELBW infants during the first days of life while on respiratory support suggesting impaired autoregulation [6-8]. Doppler studies before and after head rotation have demonstrated decreased superior sagittal sinus blood flow velocity, increased intracranial pressure $[9,10]$, as well as increased cerebral blood volume $[6,7]$ potentially placing stress on fragile germinal matrix vessels. A reduction in cerebral venous pressure has been associated with elevated midline head positioning thought to be the result of improved hydrostatic drainage from the brain [9-11]. Other studies have shown improvement in oxygenation and hypoxemic events with elevated head positioning [12, 13].

Elevated midline head positioning of preterm infants has been included in published PIVH prevention bundles or "Best Practice Guidelines" [14, 15]. However, tilting maneuvers influence not only cerebral hemodynamics but also heart rate, blood pressure and mechanics of ventilation [16]. A decrease in regional cerebral oxygenation has been documented in preterm infants during kangaroo care using near-infrared spectroscopy [17], and a decrease in cardiac output with a simultaneous increase in systemic vascular resistance was shown in late-preterm infants placed in the prone position [18]. These latter effects have not been extensively studied in a prospective randomized study.

The primary goal of this study was to determine if maintaining ELBW infants in an elevated midline head position (ELEV- supine, head of bed elevated 30 degrees, head kept in midline) versus standard head positioning (FLAT-flat supine, head turned 180 degrees every $4 \mathrm{~h}$ ) during the first 4 days of life would decrease in the incidence of PIVH. The secondary goal of the study was to determine the safety of elevated midline head positioning by comparing indices of cardiopulmonary function between the two groups.

\section{Methods}

\section{Subjects}

In this prospective randomized study, we obtained written informed consent from parents to enroll ELBW infants (birth weight $<1000$ grams) admitted to the neonatal intensive care unit at the Children's Hospital of The King's Daughters in Norfolk, Virginia. The study was approved by the Institutional Review Board (Protocol number 12-08-FB0223) at Eastern Virginia Medical School. In order to best detect an effect of position change on the occurrence of PIVH, only infants that could be randomized, placed into FLAT or ELEV position, and undergo an admission cranial ultrasound (US) within $4 \mathrm{~h}$ of birth were included in the study. Infants with congenital anomalies were excluded.

\section{Technique of maintaining elevated midline position}

ELBW infants were admitted to the neonatal intensive care unit and placed supine in the incubator (Giraffe ${ }^{\mathrm{TM}}$ Incubator, Ohmeda Medical, GE Healthcare, Chicago, Illinois) while umbilical lines were placed and radiographs obtained. Infants were randomized using a block randomization table to standard care (FLAT, supine/flat with turning of the head 90 degrees right or left every $4 \mathrm{~h}$ ) or study position (ELEV, supine, elevation of the head/body 30 degrees above the horizontal with the head maintained in the midline) for the first 4 days of life. After the fourth day all infants were placed in the flat position.

In order to maintain an elevated 30 degree position, a custom wedge-shaped frame (Fig. 1a) was prepared by our biomedical department to slide beneath the Giraffe ${ }^{\mathrm{TM}}$ incubator bed platform (Fig. 1b, c). The infants randomized to ELEV were placed in a SnuggleUp ${ }^{\mathrm{TM}}$ (Philips Medical Systems, Andover, Massachusetts) with midline head positioning maintained by gel pillows (Tarry Medical Products, Danbury, Connecticut). The endotracheal tube was supported on the incubator extension arm (Fig. 1c) 


\section{Maternal demographic and health data}

We obtained data on maternal health, pregnancy, and labor and delivery at the time of admission. Specifically, we recorded diagnoses of chronic hypertension, diabetes, HELLP syndrome, preeclampsia, preterm labor, intrapartum hemorrhage and chorioamnionitis. Maternal medications were noted, as well as evidence of fetal distress and type of delivery.

\section{Neonatal demographic, respiratory and hemodynamic data}

Gestational age, birth weight, gender, multiple gestation, Apgar scores and type of resuscitation required were noted. The time of birth, time of placement in the randomized position and admission cranial US were documented. Respiratory and hemodynamic data were collected daily for the first 4 days of life. The daily maximal $\mathrm{FiO}_{2}$, the lowest $\mathrm{pH}$, and the highest $\mathrm{pCO}_{2}$ were recorded, as well as the level of respiratory support including high frequency ventilation, conventional ventilation, continuous positive expiratory pressure and high flow nasal cannula. Arterial or capillary blood samples were obtained at regular intervals no less frequent than every $8 \mathrm{~h}$ during the 4-day period. Additionally, the number of chest radiographs, the occurrence of pneumothoraces, time to extubation, time to achieve room air ventilation, the occurrence of pulmonary hemorrhage and the incidence of BPD were documented. BPD was defined as the requirement for supplemental oxygen at 36 weeks postmenstrual age [19]. Ventilator changes were made at the discretion of the attending neonatologist.

Mean blood pressure (MBP) was measured with an indwelling umbilical arterial catheter when possible. However, when placement of an arterial line was unsuccessful, MBP was measured with a size 1 or 2 blood pressure cuff at least every $4 \mathrm{~h}$. The lowest mean blood pressure and the requirement for vasopressor support were documented. Vasopressor medications were used if a blood pressure was $<10$ th percentile for birth weight and postnatal age as determined by the attending neonatologist [20]. The presence of a hemodynamically significant PDA was assessed at least daily by clinical exam. If a significant PDA was suspected, echocardiography was performed and management was at the discretion of the attending neonatologist. Protocol variations were documented by the bedside nurse.

Infants were followed until death or discharge for the diagnoses of sepsis, urinary tract infection, necrotizing enterocolitis, intestinal perforation, retinopathy of prematurity, BPD, and length of hospital stay.

\section{Cranial ultrasound studies}

An initial cranial ultrasound exam was performed on each patient immediately after randomization and placement into the FLAT or ELEV position using a SIUI CTS-8800 S series ultrasound system (Shantou Institute of Ultrasonic Instruments Company, LTD., Shantou, China). Follow-up ultrasounds were performed daily for the first 4 days of life and on day 7. Normal scans were repeated at 4 weeks of age, abnormal scans were repeated weekly. All cranial ultrasound studies were interpreted by one of five pediatric radiologists all blinded to the randomization group. Clinical readings were used. When discrepancies in interpretation were noted, the investigators reviewed the HUS readings with the radiologists. If resolution could not be reached, the interpretation of our pediatric neuro-radiologist was used.

Grading of PIVH was done using previously described criteria [1]. An echodensity on US consistent with hemorrhage limited to the germinal matrix area was termed a Grade 1 PIVH, while blood found in the lateral cerebral ventricle but filling less than $50 \%$ of the ventricle was termed a Grade 2 PIVH. If blood distended the lateral ventricle or filled more than $50 \%$ of the ventricular volume, the lesion was termed a Grade 3 PIVH. Echodensities found in the cerebral parenchymal white matter lateral to the lateral ventricles were termed a Grade 4 PIVH or periventricular hemorraghic infarction. This latter lesion is generally associated with an ipsilateral Grade 2 or 3 PIVH. Although the more precise term is periventricular hemorrhagic infarction, for simplicity we will refer to this lesion as a Grade 4 PIVH. Cystic periventricular leukomalacia was diagnosed when prominent periventricular white matter echodensities were seen on early US studies evolving into cysts over a period of weeks.

\section{Statistical analysis}

A review of our patient data over a previous four-year period indicated an overall incidence of PIVH of $40 \%$ in our population of ELBW infants. Eighteen percent of these infants developed the more severe forms of PIVH, grade 3 and 4. A power analysis determined that for a Chi-square test with one degree of freedom, a significance level of $\alpha=$ 0.05 , and an anticipated $20 \%$ difference in the occurrence of PIVH, a total sample size of 180 infants would have $80 \%$ power to detect a statistically significant difference in the occurrence rates of PIVH between the FLAT and ELEV groups.

Block randomization was performed using SAS V9.4 (Cary, North Carolina, USA). Continuous variables were described using mean and standard deviation, and categorical variables were described using percentages. A Chisquare test was used to analyze the difference in percentage of de-escalation from a regular ventilation mode to lower modes between FLAT and ELEV groups. The Odds Ratio (OR) and 95\% CI are provided. All statistical analyses were performed using RStudio Team (2016) and SPSS (Chicago, 
IL, USA). All statistical tests were two-sided and $p<0.05$ was considered statistically significant. The effects of cofounders were considered using multivariate analyses.

\section{Results}

From October 2012 to December 2015, 354 extremely low birth weight infants were admitted to our neonatal intensive care unit, with an average gestational age of 25.5 weeks and average birth weight 728 grams. One hundred seventy were male and 184 were female. Sixty infants were one of twins and were randomized independently. Forty-nine were outborn and could not be enrolled within $4 \mathrm{~h}$ of birth, the parents of 10 infants declined enrollment, 1 was admitted at 4 days of age, and 1 infant had an omphalocele. The investigators were unavailable at the time of admission to enroll 113 of the infants.

One-hundred eighty infants were enrolled and randomized to FLAT or ELEV positioning. The FLAT group consisted of 90 infants with an average gestational age of 25.4 weeks, average birth weight of 724 grams, 54\% female, and 12 infants were one of twins. The ELEV group consisted of 90 infants with an average gestational age of 25.8 weeks, average birth weight of 745 grams, 52\% female, and 17 infants were one of twins (see Fig. 2).

The enrolled infants were placed into the randomized position and examined with the initial US by a mean age of $2.9 \mathrm{~h} \pm 1.03 \mathrm{~h}(\mathrm{SD})$, and there was no significant difference in the age at the time of admission between the two study groups $(p=0.35)$. The nursing staff documented protocol variations in 26 infants randomized to the ELEV group.

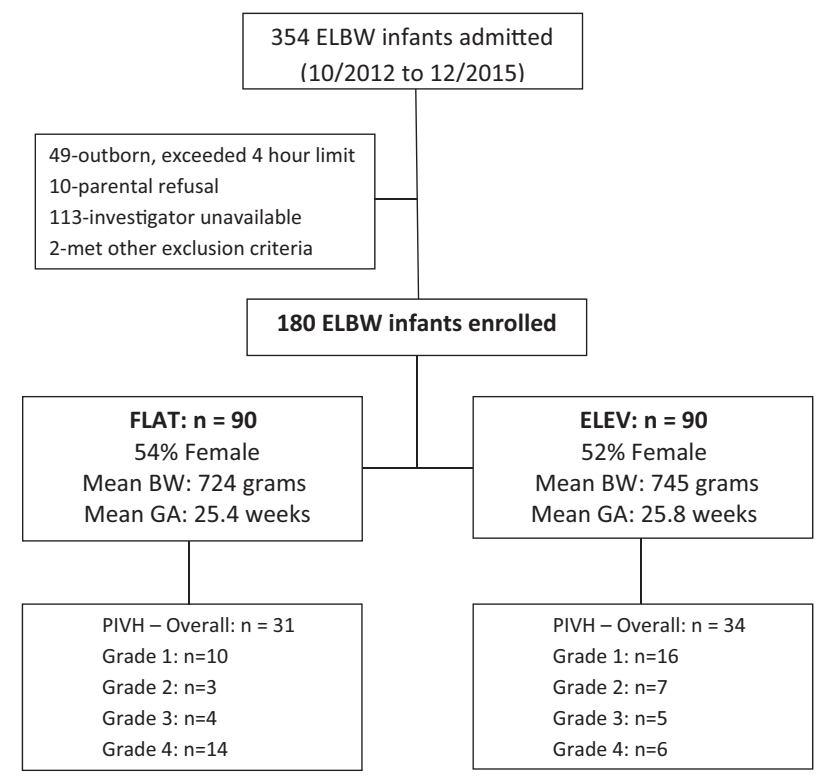

Fig. 2 Patient enrollment flowchart

49-outborn, exceeded 4 hour limit

10-parental refusal

180 ELBW infants enrolled
Table 1. Demographic data

\begin{tabular}{|c|c|c|}
\hline Head $p$ & & \\
\hline FLAT & ELEV & $\begin{array}{l}P \text { - } \\
\text { value }\end{array}$ \\
\hline
\end{tabular}

Maternal demographic data

Age, mean \pm S.D. (years)

$27.93 \pm 5.95$

$28.23 \pm 6.01$

0.73

Multiple gestation, $n(\%)$

$14(15.5)$

18 (20.0)

0.48

Chronic hypertension, $n(\%)$

Diabetes, $n(\%)$

$5(5.6)$

15 (16.7)

0.99

HELLP syndrome, $n(\%)$

$10(11.1)$

$11(12.2)$

0.19

Preeclampsia, $n(\%)$

22 (24.4)

$9(10.0)$

0.81

Perinatal bleeding, $n(\%)$

$2(2.2)$

Preterm labor, $n(\%)$

$52(57.8)$

36 (40.0)

0.026

Antepartum hemorrhage,

$n(\%)$

Placental abruption, $n(\%)$

3 (3.3)

1 (1.1)

0.62

48 (53.3)

0.46

Chorioamnionitis, $n(\%)$

$13(14.4)$

4 (4.4)

0.99

Fetal heart rate decelerations,

$n(\%)$

\begin{tabular}{|c|c|c|c|}
\hline None & $53(58.8)$ & $56(62.2)$ & 0.23 \\
\hline Variable & $21(23.3)$ & $13(14.4)$ & \\
\hline Late & $16(17.8)$ & $21(23.3)$ & \\
\hline $\begin{array}{l}\text { Prolonged rupture of } \\
\text { membranes, } n(\%)\end{array}$ & $21(23.3)$ & $9(10)$ & 0.018 \\
\hline Antenatal antibiotics, $n(\%)$ & $58(64.4)$ & $53(58.9)$ & 0.36 \\
\hline \multicolumn{4}{|c|}{ Antenatal betamethasone, $n(\%)$} \\
\hline None & $15(16.7)$ & $11(12.2)$ & 0.63 \\
\hline One dose & $17(18.9)$ & $18(20.0)$ & \\
\hline Two doses & $58(64.4)$ & $61(67.8)$ & \\
\hline Antenatal $\mathrm{MgSO}_{4}, n(\%)$ & $57(63.3)$ & $59(65.6)$ & 0.91 \\
\hline \multicolumn{4}{|l|}{ Mode of delivery, $n(\%)$} \\
\hline Vaginal & $25(27.8)$ & $18(20.0)$ & 0.23 \\
\hline Cesarean & $65(72.2)$ & $72(80.0)$ & \\
\hline \multicolumn{4}{|l|}{ Infant demographic data } \\
\hline $\begin{array}{l}\text { Gestational age (weeks), } \\
\text { mean } \pm \text { S.D }\end{array}$ & $25.39 \pm 1.64$ & $25.89 \pm 1.74$ & 0.06 \\
\hline $\begin{array}{l}\text { Birth weight (grams), } \\
\text { mean + S.D. }\end{array}$ & $\begin{array}{l}725.83 \pm \\
149.94\end{array}$ & $\begin{array}{l}7.39 .40 \pm \\
170.35\end{array}$ & 0.58 \\
\hline \multicolumn{4}{|l|}{ Size for gestational age, $n(\%)$} \\
\hline AGA & $70(77.8)$ & $64(71.1)$ & 0.36 \\
\hline IUGR $<10$ th percentile & $13(14.4)$ & $16(17.8)$ & \\
\hline IUGR $<3$ rd percentile & $7(7.8)$ & $10(11.1)$ & \\
\hline Gender, $n(\%)$ Male & $39(43.8)$ & $41(45.6)$ & 0.77 \\
\hline Female & $51(56.7)$ & $49(54.4)$ & \\
\hline Hospital, $n(\%)$ In-born & $79(87.8)$ & $83(92.2)$ & 0.61 \\
\hline Out-born & $11(12.2)$ & $7(7.8)$ & \\
\hline $\begin{array}{l}\text { Epinephrine during } \\
\text { resuscitation, } n(\%)\end{array}$ & $0(0.0)$ & $3(3.3)$ & 0.25 \\
\hline Chest compressions, $n(\%)$ & $1(1.1)$ & $4(4.4)$ & 0.37 \\
\hline Surfactant, $n(\%)$ & $84(93.3)$ & $88(97.8)$ & 0.72 \\
\hline
\end{tabular}

0.05

0.61

$10(11.1)$

23

\section{8}

36

63

\section{6}

Surfactant, $n(\%)$ 
Table 1. (continued)

\begin{tabular}{llll}
\hline & \multicolumn{2}{l}{ Head position } & \\
\cline { 2 - 4 } & FLAT & ELEV & $\begin{array}{l}P \text { - } \\
\text { value }\end{array}$ \\
\hline $\begin{array}{l}\text { Apgar score at I minute, } \\
\text { median (range) }\end{array}$ & $2(0,7)$ & $3(0,8)$ & 0.44 \\
$\begin{array}{l}\text { Apgar Score at 5 min, median } \\
\text { (range) }\end{array}$ & $6(1,9)$ & $6(1,9)$ & 0.37 \\
\hline
\end{tabular}

$S D$ standard deviation; HELLP hypertension, elevated liver function tests, low platelets; $P I H$ pregnancy induced hypertension; $A G A$ appropriate for gestational age; IUGR intrauterine growth restriction, statistically significant $\mathrm{p}$ values are in bold type

Nineteen infants had relatively brief periods of flat positioning during repeat surfactant administration (6), PICC placement (6), and reintubation (7). One infant had a respiratory deterioration associated with a pulmonary hemorrhage and one infant underwent abdominal drain placement, both were placed back in the ELEV position after stabilization. One infant was kept in the flat position when a pulmonary hemorrhage occurred on day 3 of life. Four infants were placed flat when respiratory support had to be increased to high-frequency ventilation ( 2 on day 2 of life, 2 on day 3 of life). Data from all infants were maintained in their original randomization group.

\section{Maternal and infant demographic and birth history data}

Maternal and infant demographic data are shown in Table 1. Significant differences were noted between the FLAT and ELEV groups in the number of mothers with preeclampsia (FLAT 24.4\%, ELEV 40\%, $p=0.026$ ), and prolonged premature rupture of membranes (FLAT 23.3\%, ELEV $10 \%, p=0.018)$. Multivariate analysis of maternal preeclampsia and prolonged rupture of membranes showed no significant effect on outcomes. There were no significant differences in the infant data between the two groups.

\section{Respiratory data}

Data from blood gas measurements are shown in Table 2, and respiratory support and respiratory outcome data are shown in Table 3. The infants in the ELEV group had a significantly lower maximal $\mathrm{FiO}_{2}$ requirement during the first $24 \mathrm{~h}$ than infants in the FLAT group $(p=0.009)$, however the ELEV group had significantly higher maximal $\mathrm{pCO}_{2}$ levels on days $2(p=0.002)$ and $3(p=0.001)$. The increase in the $\mathrm{pCO}_{2}$ levels are reflected in the higher levels of respiratory support required. As shown in Table 3, a significant escalation in respiratory support to high frequency ventilation was noted for infants in the ELEV group during the first 4 days of life. The odds of an increase in
Table 2. Respiratory-Blood gas data

\begin{tabular}{|c|c|c|c|}
\hline & \multicolumn{3}{|c|}{ Head position } \\
\hline & FLAT & ELEV & $p$-value \\
\hline Day 1 (number of infants) & $(n=90)$ & $(n=90)$ & \\
\hline${ }^{\mathrm{a}} \mathrm{Maximum} \mathrm{FiO2}$ & $46.0(26.49)$ & $37.5(15.97)$ & 0.009 \\
\hline${ }^{\mathrm{a}}$ Lowest $\mathrm{pH}$ & $7.162(0.16)$ & $7.186(0.10)$ & 0.16 \\
\hline${ }^{\mathrm{a}}$ Highest pCO2 & $60.4(19.94)$ & $59.0(14.73)$ & 0.40 \\
\hline Day 2 (number of infants) & $(n=85)$ & $(n=89)$ & \\
\hline${ }^{\mathrm{a}}$ Maximum $\mathrm{FiO} 2$ & $38.5(22.44)$ & $36.9(19.56)$ & 0.68 \\
\hline${ }^{\mathrm{a}}$ Lowest $\mathrm{pH}$ & $7.169(0.12)$ & $7.147(0.12)$ & 0.32 \\
\hline${ }^{\mathrm{a}}$ Highest pCO2 & $58.6(16.51)$ & $68.1(22.58)$ & 0.002 \\
\hline Day 3 (number of infants) & $(n=84)$ & $(n=89)$ & \\
\hline${ }^{\mathrm{a}}$ Maximum FiO2 & $34.4(19.65)$ & $37.5(20.51)$ & 0.28 \\
\hline aLowest $\mathrm{pH}$ & $7.158(0.13)$ & $7.149(0.09)$ & 0.56 \\
\hline${ }^{\mathrm{a}}$ Highest $\mathrm{pCO} 2$ & $56.7(13.11)$ & $64.0(15.75)$ & 0.001 \\
\hline Day 4 (number of infants) & $(n=82)$ & $(n=89)$ & \\
\hline${ }^{\mathrm{a}}$ Maximum FiO2 & $31.4(16.39)$ & $35.7(18.25)$ & 0.09 \\
\hline${ }^{\mathrm{a}}$ Avg. Lowest $\mathrm{pH}$ & $7.176(0.11)$ & $7.169(0.09)$ & 0.66 \\
\hline${ }^{\text {a}}$ Highest pCO2 & $54.3(14.30)$ & $58.5(13.64)$ & 0.07 \\
\hline
\end{tabular}

${ }^{\mathrm{a}}$ Average ( \pm Standard deviation); $\mathrm{FiO} 2(\%), \mathrm{pCO} 2$ reported in $\mathrm{mmHg}$, statistically significant $\mathrm{p}$ values are in bold type

support in the ELEV group was 1.3 times higher than the FLAT group, OR $=1.33$, CI $(1.07,1.65),(p=0.01)$. Significantly higher mean airway pressures were required by infants on high frequency ventilation randomized to the FLAT group on day $1(p=0.023)$. By day 4 , ELEV infants on conventional ventilation required significantly greater mean airway pressure than FLAT infants on similar type of ventilator support $(p=0.027)$.

\section{Hemodynamic data}

Hemodynamic data is shown in Table 4. On day 1 and day 3 , the lowest mean blood pressures were significantly higher in the ELEV group over the FLAT group (day 1, 20.0 vs. $22.2 \mathrm{mmHg}, p=0.003$ ) (day $3,26.7$ vs. $28.8 \mathrm{mmHg}$, $p=0.03$ ). There were no significant differences noted in dopamine, epinephrine or steroid administration, as well as the incidence or treatment of patent ductus arteriosus between the two groups.

\section{Complications of preterm birth}

There were no significant differences in the incidence of sepsis, urinary tract infection, necrotizing enterocolitis, intestinal perforation, retinopathy of prematurity and length of hospital stay between the FLAT and ELEV groups (Table 5). However, infants randomized to the ELEV group were significantly more likely to survive to discharge (FLAT 76\%, ELEV 88\%, $p=0.033$ ). 
Table 3. Respiratory support and respiratory outcome data

\begin{tabular}{|c|c|c|c|c|c|c|c|c|c|}
\hline & \multicolumn{9}{|c|}{ Head position } \\
\hline & \multicolumn{4}{|l|}{ FLAT } & \multicolumn{4}{|l|}{ ELEV } & \multirow[t]{2}{*}{$p$-value } \\
\hline & Day 1 & Day 2 & Day 3 & Day 4 & Day 1 & Day 2 & Day 3 & Day 4 & \\
\hline Number of infants & 90 & 85 & 84 & 82 & 90 & 89 & 89 & 89 & \\
\hline \multirow{2}{*}{$\begin{array}{l}\text { Number on HFV } \\
\text { MAP (SD) }\end{array}$} & 17 & 21 & 23 & 24 & 8 & 19 & 24 & 27 & \\
\hline & $\begin{array}{l}11.5 \\
(1.8)\end{array}$ & $\begin{array}{l}10.7 \\
(2.1)\end{array}$ & $\begin{array}{l}10.8 \\
(1.7)\end{array}$ & $\begin{array}{l}10.8 \\
(1.5)\end{array}$ & $9.9(0.6)$ & $\begin{array}{l}10.6 \\
(2.2)\end{array}$ & $\begin{array}{l}11.3 \\
(4.3)\end{array}$ & $\begin{array}{l}11.3 \\
(4.2)\end{array}$ & $\begin{array}{l}\text { Day 1-0.023, Day 2-0.87, } \\
\text { Day 3-0.59, Day 4-0.57 }\end{array}$ \\
\hline \multirow{2}{*}{$\begin{array}{l}\text { Number on CV } \\
{ }^{\mathrm{a}} \mathrm{MAP} \text { (SD) }\end{array}$} & 72 & 41 & 33 & 29 & 81 & 53 & 47 & 45 & \\
\hline & $8.0(1.0)$ & $7.6(1.7)$ & $8.0(1.5)$ & $7.4(1.1)$ & $8.3(2.3)$ & $8.2(3.0)$ & $8.3(1.5)$ & $8.0(1.1)$ & $\begin{array}{l}\text { Day } 1-0.32 \text {, Day } 2-0.28, \\
\text { Day } 3-0.29, \text { Day } \mathbf{4 - 0 . 0 2 7}\end{array}$ \\
\hline Number on NCPAP & 0 & 19 & 17 & 14 & 1 & 15 & 15 & 12 & \\
\hline Number on HFNC & 1 & 4 & 11 & 15 & 0 & 2 & 3 & 5 & \\
\hline Number of XRs (SD) & \multicolumn{4}{|l|}{$5.6(3.14)$} & \multicolumn{4}{|l|}{$6.0(2.93)$} & 0.36 \\
\hline Pneumothorax (\%) & \multicolumn{4}{|l|}{$4(4 \%)$} & \multicolumn{4}{|l|}{$2(2 \%)$} & 0.68 \\
\hline $\begin{array}{l}\text { Age at extubation median } \\
\text { (range) }\end{array}$ & \multicolumn{4}{|c|}{25 days $(0,372)$} & \multicolumn{4}{|c|}{27 days $(0,434)$} & 0.36 \\
\hline Age to room air (SD) & \multicolumn{4}{|c|}{54 days $(27.30)$} & \multicolumn{4}{|c|}{62 days (42.90) } & 0.23 \\
\hline Pulmonary hemorrhage (\%) & \multicolumn{4}{|l|}{$5(6 \%)$} & \multicolumn{4}{|l|}{$6(7 \%)$} & 0.99 \\
\hline $\mathrm{BPD}(\%)$ & \multicolumn{4}{|l|}{$30(43 \%)$} & \multicolumn{4}{|l|}{$31(39 \%)$} & 0.68 \\
\hline
\end{tabular}

$H F V$ high frequency ventilation, $M A P$ mean airway pressure, $C V$ conventional ventilation, $N C P A P$ nasal continuous positive airway pressure, $H F N C$ high flow nasal cannula, XR radiographs, $S D$ standard deviation, $\%$ percent, $B P D$ bronchopulmonary dysplasia, statistically significant $\mathrm{p}$ values are in bold type

a average + standard deviation

Table 4. Hemodynamic data

\begin{tabular}{llll}
\hline & \multicolumn{3}{l}{ Head position } \\
\cline { 2 - 4 } & FLAT & ELEV & $p$-value \\
\hline Lowest mean blood pressure Average $m m H g(S D)$ & \\
Day 1 & $20.0(5.02)$ & $22.2(5.01)$ & $\mathbf{0 . 0 0 3}$ \\
Day 2 & $25.7(7.44)$ & $27.3(6.63)$ & 0.11 \\
Day 3 & $26.7(7.99)$ & $28.8(6.76)$ & $\mathbf{0 . 0 3}$ \\
Day 4 & $28.4(6.45)$ & $30.1(7.13)$ & 0.07 \\
Dopamine during the first 4 days & $43(48 \%)$ & $35(39 \%)$ & 0.29 \\
Epinephrine in the first 4 days & $20(22 \%)$ & $15(17 \%)$ & 0.45 \\
Steroids in the first 4 days & $19(21 \%)$ & $15(17 \%)$ & 0.57 \\
Patent ductus arteriosus & $48(53 \%)$ & $55(61 \%)$ & 0.37 \\
Indocin (one course) & $10(11 \%)$ & $20(22 \%)$ & 0.07 \\
Indocin (multiple courses) & $8(9 \%)$ & $6(7 \%)$ & 0.78 \\
Ligation & $16(18 \%)$ & $18(20 \%)$ & 0.85 \\
\hline SD standard
\end{tabular}

$S D$ standard deviation, statistically significant $\mathrm{p}$ values are in bold type

\section{Cranial ultrasound results}

Five infants in the FLAT group had a PIVH on admission, 3 with grade 1 PIVH, 1 with grade 2 and 1 with grade 3 PIVH. Nine infants in the FLAT group expired during the first 4 days of life (mean 2.2 days), all received at least
Table 5. Complications of preterm birth

\begin{tabular}{llll}
\hline & \multicolumn{2}{l}{ Head position } & \\
\cline { 2 - 4 } Outcome & FLAT & ELEV & $p$-value \\
\hline Sepsis & $23(26 \%)$ & $22(24 \%)$ & 0.99 \\
UTI & $12(13 \%)$ & $11(12 \%)$ & 0.99 \\
NEC (suspected) & $2(2 \%)$ & $3(3 \%)$ & 0.99 \\
NEC (medical treatment) & $8(9 \%)$ & $9(10 \%)$ & 0.99 \\
NEC (surgical treatment) & $2(2 \%)$ & $5(6 \%)$ & 0.44 \\
Intestinal perforation (no & $4(4 \%)$ & $8(9 \%)$ & 0.37 \\
NEC) & & & \\
ROP requiring treatment & $11(12 \%)$ & $8(9 \%)$ & 0.63 \\
Survival to discharge & $68(76 \%)$ & $79(88 \%)$ & $\mathbf{0 . 0 3 3}$ \\
Average duration of & 92 days & 109 days & 0.11 \\
hospitalization (SD) & $(68.8)$ & $(69.9)$ & \\
\hline
\end{tabular}

UTI urinary tract infection, NEC necrotizing enterocolitis, ROP retinopathy of prematurity, $S D$ standard deviation, statistically significant $\mathrm{p}$ values are in bold type

one US. Seven were normal, 1 had a Grade 3 PIVH and 1 had a Grade 4 PIVH. These early deaths were associated with severe respiratory disease. Thirteen infants in the FLAT group expired after 4 days (mean 30.2 days), 4 had normal US studies, 2 had Grade 2 PIVH, 1 had a Grade 3 PIVH and 6 had Grade 4 PIVH. The later 


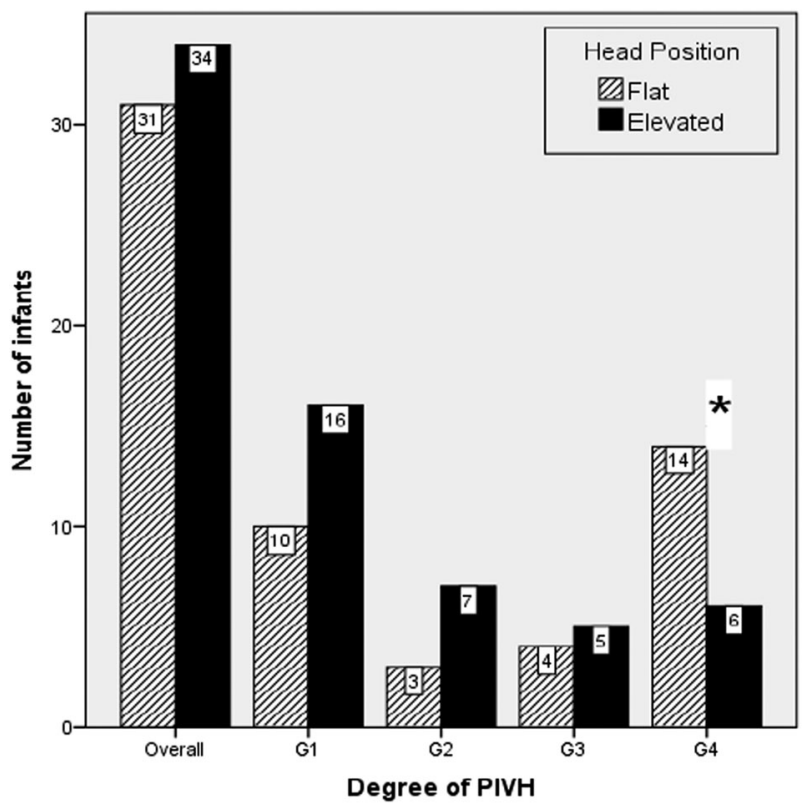

Fig. 3 This graph shows the number of infants in the FLAT and ELEV groups with the various grades of PIVH, G4 refers to periventricular hemorrhagic infarction (G1 $p=0.34, \mathrm{G} 2 p=0.23$, G3 $p=0.72$ and $\left.\mathrm{G} 4 p=0.036^{*}\right)$

deaths were associated with other complications of prematurity: NEC (5), sepsis (2), UTI (1), pulmonary hemorrhage (2), and respiratory failure without evidence of infection (3). Some degree of PIVH was noted in 31 of the 90 infants $(34 \%)$ randomized to the FLAT group. The number of infants with each grade of PIVH is shown in Fig. 3.

Six infants in the ELEV group had a PIVH on admission, 3 with grade 1 PIVH and 3 with grade 2 PIVH. Two infants in the ELEV group expired during the first 4 days of life (both on day 3), both early deaths were associated with respiratory failure and one had a pulmonary hemorrhage. One of these infants had a Grade 4 PIVH, the other had a normal US. Nine infants in the ELEV group expired after 4 days (mean 38.9 days), 2 had a normal US, 2 had Grade 1 PIVH, 1 had a grade 2 PIVH, 2 had grade 3 PIVH and 2 had grade 4 PIVH. These deaths were associated with sepsis (2), NEC (4), complications of twin to twin transfusion syndrome (2), and 1 with respiratory failure without evidence of infection. Some degree of PIVH was noted in 34 of the 90 infants $((37.8 \%)$ randomized to the ELEV group. Although some infants developed subtle white matter echodensities possibly consistent with evolving periventricular leukomalacia, none developed cystic lesions detectable with US during the study period.

The incidence of PIVH for the ELEV and FLAT groups is shown in Fig. 3. There was no significant difference between the FLAT and the ELEV group for grades 1, 2 and 3, however the ELEV group showed a significant decrease in the incidence of grade 4 PIVH $(p=0.036)$. Progression of PIVH to a more severe grade that was documented by US during the course of the study occurred in $12(39 \%)$ of the FLAT group and $5(15 \%)$ of the ELEV group ( $p=0.033$, OR $=0.27$, CI 0.08-0.90). These relationships persisted when infants with PIVH on admission were excluded.

\section{Discussion}

In this study we found that managing ELBW infants in a position of head elevation to 30 degrees with the head maintained in the midline is safe, and may even be beneficial in term of lower $\mathrm{FiO} 2$ requirement, improved mean blood pressure and potentially improved survival. Despite higher pCO2 levels and increased requirement for highfrequency ventilation in the first 4 days of life in the ELEV group, there was no significant difference in the incidence of BPD or the length of hospital stay between the two groups. Although the overall incidence of PIVH was similar between the two study groups, infants in the ELEV group demonstrated a lower likelihood of a progression to the more severe grades of PIVH, and most importantly, they developed significantly fewer grade 4 hemorrhages. The overall incidence of PIVH and the distribution of various grades of PIVH found in this study are similar to previously published NICHD data that reported an overall incidence of PIVH in ELBW infant of $31 \%$ and a $9 \%$ incidence of grade 4 PIVH [21].

The choice of using a 30 degree head elevation in this study was somewhat arbitrary. Previous reports have documented potentially beneficial changes in intracranial pressure and cerebral oxygenation in infants with head elevation of 15 to 45 degrees [9-14, 17]. We wanted to elevate the head of the bed more than the 12 degrees the Giraffe $^{\circledR}$ incubators will easily allow, and nursing staff felt they would not have enough space to perform daily care if the head of the bed were raised to 45 degrees. The effects of midline head positioning should be studied separately from head elevation, however since data does exist that both may be helpful, we chose to do both.

There was a significantly greater percentage of survival to discharge in the infants randomized to the ELEV group (FLAT 76\%, ELEV 88\%, $\mathrm{p}=0.033$ ). Previous studies from the NICHD Neonatal Research Network report survival rates of ELBW infants born at 25 weeks gestation (the approximate average gestational age of our cohort of infants) of $72 \%$ ranging from 50 to $90 \%$ across all centers [21]. It has been suggested that providing respiratory support in an upright position might lessen ventilator associated pneumonia [22, 23]. This potential benefit of upright 
ventilation could enhance survival of ELBW infants randomized to the ELEV group. Infants randomized to the ELEV group had insignificant trends for greater gestational age and birth weight, but more often required vigorous resuscitation in the delivery room. Survival may have been influenced by significantly more preeclampsia [24] and less prolonged rupture of membranes in mothers of infants randomized to the ELEV group.

One weakness of our study was the number of infants with protocol variations. Although most of the protocol variations were brief, some infants randomized to the ELEV group remained flat for the final 1 to 2 days of the 4-day period ELEV period possibly altering the study results. In addition, recording only the extremes of blood gas parameters, i.e. the greatest $\mathrm{FiO}_{2}$ and $\mathrm{pCO}_{2}$ and the lowest $\mathrm{pH}$ during a $24 \mathrm{~h}$ period does not fully reflect the overall respiratory health of the infant.

The exclusive use of US as our neuroimaging technique is another weakness of our study. While hemorrhage associated with PIVH is easily seen on US, the ischemic white matter of non-cystic periventricular leukomalacia is not. The use of MRI could have provided more information on the incidence of ischemic cerebral white lesions and probably detected lesions we missed with US. The addition of Doppler flow studies and near-infrared spectroscopy could have shed light on alterations in cerebral blood flow and cerebral oxygenation associated with changes in head position and suggested potential mechanisms of mitigating brain injury

We attempted to study the most vulnerable infants during their most critical time period for the occurrence of PIVH [1]. Although we did not demonstrate a significant difference in the overall incidence of PIVH or a difference in the individual of grades 1, 2 and 3 PIVH, infants managed in the ELEV position developed significantly fewer grade 4 hemorrhages, the lesion most responsible for the high rate of neurologic impairments seen in the ELBW population [1]. In addition, a decrease in the likelihood of progression to a more severe grade of PIVH was noted in the ELEV group.

This study supports the concept that significant changes in cerebrovascular circulation are associated with position changes of ELBW newborns and elevated midline positioning of the head may have important effects on cerebral blood flow, autoregulation, cerebral venous pressure, and cerebral venous drainage, with the end result of fewer serious cerebral hemorrhages. We think these results are encouraging and are worthy of further study.

Acknowledgements The authors thank the excellent, dedicated nursing staff and the respiratory therapy staff of the Children's Hospital of The King's Daughters Neonatal Intensive Care Unit for their support and assistance.

\section{Compliance with ethical standards}

Conflict of interest The authors declare that they have no conflict of interest.

\section{References}

1. Volpe JJ. Intracranial hemorrhage: Germinal matrixintraventricular hemorrhage of the premature infant. In: Neurology of the Newborn (5th edition). Philadelphia, PA: Saunders Elsevier, 2008, pp 517-88.

2. Ballabh P. Intraventricular hemorrhage in premature infants: mechanism of disease. Pediatr Res. 2010;67:1-8.

3. Gilmore MM, Stone BS, Shepard JA, Czosnyka M, Easley RB, Brady KM. Relationship between cerebrovascular dysautoregulation and arterial blood pressure in the premature infant. $\mathbf{J}$ Perinatol. 2011;31:722-9.

4. Limperopoulos C, Gauvreau KK, O'Leary H, Moore M, Bassan H, Eichenwald EC, Soul JS, Ringer SA, Di Salvo DN, du Plessis A. Cerebral hemodynamic changes during intensive care of preterm infants. Pediatrics. 2008;122:e1006-13.

5. Bembich S, Oretti C, Travan L, Clarici A, Massaccesi S, Demarini $\mathrm{S}$. Effect of prone and supine position on cerebral blood flow in preterm infants. J Pediatr. 2012;160:162-4.

6. Pichler G, Urlesberger B, Schmolzer G, Muller W. Effect of tilting on cerebral haemodynamics in preterm infants with periventricular leucencephalomalacia. Acta Paediatr. 2004;93:70-75.

7. Pellicer A, Gaya F, Madero R, Quero J, Cabanas F. Noninvasive continuous monitoring of the effects of the head position on brain hemodynamics in ventilated infants. Pediatrics. 2002;109:434-40.

8. Ancora G, Maranella E, Aceti A, Pierantoni L, Grandi S, Corvaglia $\mathrm{L}$, et al. Effect on posture on brain hemodynamics in preterm newborns not mechanically ventilated. Neonatology. 2010; 97:212-7.

9. Cowan F, Thoresen M. Changes in superior sagittal sinus blood velocities due to postural alterations and pressure on the head of the newborn infant. Pediatrics. 1985;75:1038-47.

10. Emery JR, Peabody JL. Head position affects intracranial pressure in newborn infants. Pediatrics. 1983;103:950-3.

11. Goldberg RN, Joshi A, Moscoso P, Castillo T. The effect of head position on intracranial pressure. Crit Care Med. 1983;11:428-30.

12. Dellagrammaticas HD, Kapetenakis J, Papadimitriou M, Kourakis G. Effect of body tilting on physiological functions in stable very low birthweight neonates. Arch Dis Child. 1991;66:429-32.

13. Jenni OG, von Siebenthal K, Wolf M, Keel M, Duc G, Bucher HU. Effect of nursing in the head elevated tilt position $\left(15^{\circ}\right)$ on the incidence of bradycardic and hypoxemic episodes in preterm infants. Pediatrics. 1997;100:622-5.

14. Carteaux P, Cohen H, Check J, George J, McKinley P, Lewis W, et al. Evaluation and development of potentially better practices for the prevention of brain hemorrhage and ischemic brain injury in very low birth weight infants. Pediatrics. 2003;111:e489-96.

15. Malusky S, Donze A. Neutral head positioning in premature infants for intraventricular hemorrhage prevention: an evidencebased review. Neonatal Netw. 2011;30:381-96.

16. Thoresen M, Cowan F, Walloe L. Cardiovascular responses to tilting in healthy newborns babies. Early Human Dev. 1991;26:213-22.

17. Schrod L, Walter J. Effect of head-up body tilt position on autonomic function and cerebral oxygenation in preterm infants. Biol Neonate. 2002;81:255-25.

18. Ma M, Noori S, Maarek J-M, Holschneider DP, Rubinstein EH, Seri I. Prone positioning decreases cardiac output and increases 
systemic vascular resistance in neonates. J Perinatol. 2015;35: 424-7.

19. Jobe A, Bancalari E. Bronchopulmonary dysplasia. Am J Respir Crit Care Med. 2001;163:1723-9.

20. Watkins AMC, West CR, Cooke RWI. Blood pressure and cerebral hemorrhage and ischemia in very low birth weight infants. Early Hum Dev. 1989;19:103-10.

21. Stoll BJ, Hansen NI, Bell EF, Shankaran S, Laptook AR, Walsh $\mathrm{MC}$, et al. Neonatal outcomes of extremely preterm infants from the NICHD Neonatal Research Network. Pediatrics. 2010;126:443-55.
22. Polin RA, Denson S, Brady MT. and the Committee on the Fetus and Newborn and the Committee on Infectious Disease. Clinical report: strategies for prevention of health care-associated infections in the NICU. Pediatrics. 2012;149:e1085-93.

23. Garland JS. Ventilator-associated pneumonia in neonates: an update. NeoReviews. 2014;15:225-35.

24. Kuban K, Leviton A, Pagano M, Fenton T, Strassfield R, Wolff M. Maternal toxemia is associated with reduced incidence of germinal matrix hemorrhage in premature infants. J Child Neurol. 1992;7:70-76. 